\title{
Thermomechanical response of Large Hadron Collider collimators to proton and ion beam impacts
}

\author{
Marija Cauchi* \\ CERN, Geneva, Switzerland and University of Malta, Msida, Malta
}

\author{
R. W. Assmann, ${ }^{\dagger}$ A. Bertarelli, F. Carra, ${ }^{\ddagger}$ F. Cerutti, L. Lari, ${ }^{\S}$ and S. Redaelli \\ CERN, Geneva, Switzerland \\ P. Mollicone and N. Sammut \\ University of Malta, Msida, Malta
}

(Received 15 February 2015; published 9 April 2015)

\begin{abstract}
The CERN Large Hadron Collider (LHC) is designed to accelerate and bring into collision high-energy protons as well as heavy ions. Accidents involving direct beam impacts on collimators can happen in both cases. The LHC collimation system is designed to handle the demanding requirements of high-intensity proton beams. Although proton beams have 100 times higher beam power than the nominal LHC lead ion beams, specific problems might arise in case of ion losses due to different particle-collimator interaction mechanisms when compared to protons. This paper investigates and compares direct ion and proton beam impacts on collimators, in particular tertiary collimators (TCTs), made of the tungsten heavy alloy INERMET $^{\circledR} 180$. Recent measurements of the mechanical behavior of this alloy under static and dynamic loading conditions at different temperatures have been done and used for realistic estimates of the collimator response to beam impact. Using these new measurements, a numerical finite element method (FEM) approach is presented in this paper. Sequential fast-transient thermostructural analyses are performed in the elastic-plastic domain in order to evaluate and compare the thermomechanical response of TCTs in case of critical beam load cases involving proton and heavy ion beam impacts.
\end{abstract}

DOI: 10.1103/PhysRevSTAB.18.041002

PACS numbers: 29.27.-a, 07.05.Tp, 79.20.Ap

\section{INTRODUCTION}

The Large Hadron Collider (LHC) operates with proton as well as heavy ion beams [1]. Collisions between beams of fully stripped lead $\left({ }^{208} \mathrm{~Pb}^{82+}\right)$ ions have been successfully carried out during the first years of operation of the LHC $[2,3]$. Moreover, the success of the first two $\mathrm{Pb}-\mathrm{Pb}$ runs led the experiments to request a proton-ion run [4], as a first extension to the LHC design, for the last exploitation period before the first long shutdown of the LHC. The main beam parameters for protons and heavy ions are listed in Table I. While the major hardware systems of the LHC ring are compatible with both proton and heavy ion operation, the physics of the ion beam's interaction with matter varies qualitatively and quantitatively from that of the proton beam, resulting in different beam dynamics and performance limits for the two types of beam [1].

"marija.cauchi@cern.ch

†Present address: DESY, Germany.

Also at Politecnico di Torino, Turin, Italy.

${ }^{\S}$ Also at IFIC (CSIC-UV), Valencia, Spain.

Published by the American Physical Society under the terms of the Creative Commons Attribution 3.0 License. Further distribution of this work must maintain attribution to the author(s) and the published article's title, journal citation, and DOI.
With a stored proton beam energy of $362 \mathrm{MJ}$ (Table I), the two counterrotating LHC beams (Beam 1 and Beam 2) are highly destructive. As the bunches rotate within the LHC ring, particles at the edges of the spatial distribution tend to escape from the proper trajectory and form a beam halo. Beam losses can cause quenches of the superconducting (SC) magnets as well as material damage $[5,6]$. Therefore, losses must be tightly controlled and

TABLE I. Design parameters for the LHCs proton $(\mathrm{p})$ and lead ion $\left({ }^{208} \mathrm{~Pb}^{82+}\right)$ beams in collision conditions. The luminosity $\mathcal{L}$ is a measure of the number of collisions that can be produced at the experiments and the $\beta^{*}$ refers to the optical amplitude function $\beta$ at the collision points [1].

\begin{tabular}{lcc}
\hline \hline Particle & $\mathrm{p}$ & ${ }^{208} \mathrm{~Pb}^{82+}$ \\
\hline Energy/nucleon [TeV] & 7 & 2.759 \\
Number of bunches $k_{b}$ & 2808 & 592 \\
Particles/bunch & $1.15 \times 10^{11}$ & $7 \times 10^{7}$ \\
Transverse normalized & 3.75 & 1.50 \\
$\quad$ emittance $(1 \sigma)[\mu \mathrm{m}]$ & & \\
RMS momentum spread $\left\langle\delta_{p}^{2}\right\rangle^{1 / 2}$ & $1.13 \times 10^{-4}$ & $1.10 \times 10^{-4}$ \\
Stored energy per beam $[\mathrm{MJ}]$ & 362 & 3.81 \\
Design luminosity $\mathcal{L}\left[\mathrm{cm}^{-2} \mathrm{~s}^{-1}\right]$ & $10^{34}$ & $10^{27}$ \\
Horizontal and vertical $\beta^{*}[\mathrm{~m}]$ & 0.55 & 0.50 \\
\hline \hline
\end{tabular}


the machine aperture must be well protected. These issues established the need for the development of a powerful collimation system [7] to protect the accelerator against unavoidable regular and irregular beam losses, as well as to ensure the proper functionality of the LHC.

The LHC collimation system has primarily been optimized for proton operation; however, it has also been used during the heavy-ion runs [8,9]. Although the stored energy of the nominal ion beam is only $1 \%$ of that of the nominal proton beam (Table I), it is important to study ion collimation because of the different characteristics of the beam-matter interaction processes.

The design philosophy of the collimation system is based on a multi-staged beam cleaning approach. The advantages of such an approach include a substantially small fraction of particles escaping from the collimators and a heat load on the collimators that is distributed over a larger number of devices. The collimation system used for the LHC Run 1 (2010-2013) consists of 108 collimators and absorbers, of which 100 are movable collimators installed in seven out of eight LHC interaction regions (IRs) as well as in the transfer lines. Moreover, collimators are installed in different configurations (horizontal, vertical, and skew) all around the ring, thus ensuring complete cleaning of the beam halo all around the particle beam axis (Fig. 1).

There are two dedicated collimation insertions in the LHC (IR3 and IR7) in which primary collimators (TCPs) and secondary collimators (TCSs) intercept the largest fraction of the beam halo and are thus made of a carbon fiber composite (CFC) to ensure high robustness. The socalled active absorbers (TCLAs) are then meant to catch tertiary halo particles scattered out of the TCSs as well as showers from upstream collimators. The TCLAs are made of a tungsten heavy alloy in order to stop as much as

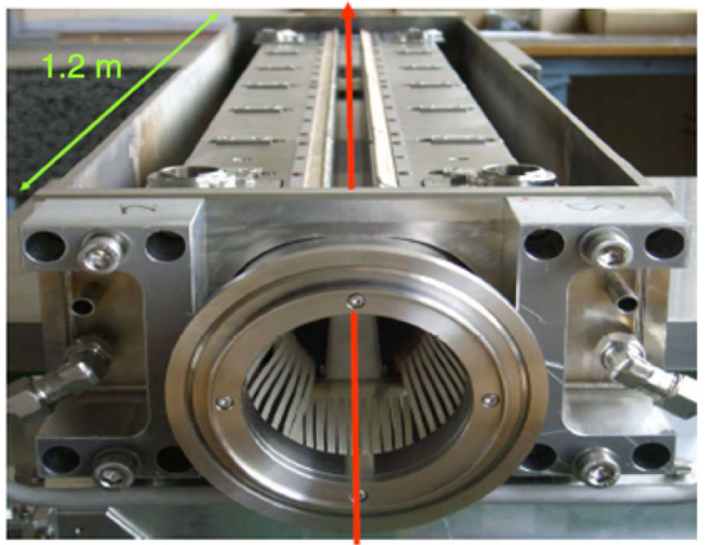

362 MJ proton beam

FIG. 1. A horizontal LHC collimator. View into the open vacuum tank of the collimator during production. The beam trajectory is shown in red, with the left and right jaw assemblies centered with respect to the closed beam orbit. The jaws lie inside the casing, on either side of the beam, with the radio-frequency fingers showing in the foreground [10]. possible of the incoming energy. However, they are not as robust as CFC collimators and thus they should never intercept primary beam losses.

In addition to these two LHC collimation insertions, there are also collimators in most other IRs. In particular, there are tertiary collimators (TCTs) that are built with the same design and materials as TCLAs. However, TCTs are installed about $150 \mathrm{~m}$ upstream of the collision points at all experiments in order to intercept the tertiary halo close to the particle physics experiments and the sensitive triplet magnets. In fact, TCTs are essential for decreasing the experimental background [11] and also for providing passive machine protection to the critical SC magnets [12].

In the worst accident case corresponding to an asynchronous trigger of the beam dumping system [13], one or more high energy density bunches might directly impact a collimator with possible serious consequences. Even though the machine configurations [14] are chosen to minimize this risk in a way that it can only occur in case several combined failures occur at the same time [15], it is important to understand the implications of such an event on a TCT. This study focuses on TCTs since they are the first metallic collimators exposed to high risks of damage in case of an asynchronous beam dump and they are essential for the protection of the downstream SC magnets. Similar studies have already been carried out for protons $[16,17]$ and the developed finite element method (FEM) approach has been successfully validated in [18].

This paper presents a numerical FEM approach in order to evaluate and compare the thermomechanical response of TCTs in case of critical beam load cases involving proton and heavy ion beam impacts. It first gives a brief overview of the physical interaction processes occurring when both particle types traverse the collimator material, followed by a description of the asynchronous beam dump accident scenario. The performed numerical analysis is then explained in detail, including also new recent measurements of the mechanical behavior of the collimator jaw insert material, INERMET ${ }^{\circledR} 180$. Thermal and structural results are presented for both proton and ion beam cases and a comparison of the thermomechanical response of TCTs in these two cases is discussed.

\section{BEAM-MATTER INTERACTION PROCESSES}

Beam-matter interaction processes are important both in terms of the local heat deposition inside the material as well as the resulting secondary scattering processes that occur. In fact, the final energy deposition in the material is, to a large extent, due to the electromagnetic shower which is developed together with the hadronic shower initiated by nuclear inelastic reactions [19]. A short review of the passage of charged particles through matter can be found in [20].

The physics of the particle-matter interactions for heavy ions is qualitatively different from that for protons (Table II) and thus the ion-matter interactions in collimators result in 
TABLE II. ${ }^{208} \mathrm{~Pb}^{+}$ion-matter interactions in comparison with proton-matter interactions. ECPP and EMD stand for electron capture pair production and electromagnetic dissociation respectively. Values are for particle impact on graphite [1].

\begin{tabular}{lcccc}
\hline \hline Physics process & p injection & p collision & ${ }^{208} \mathrm{~Pb}^{+}$injection & ${ }^{208} \mathrm{~Pb}^{+}$collision \\
\hline Energy per nucleon $[\mathrm{TeV}]$ & 0.44 & 7 & 0.1774 & 2.759 \\
Ionization energy loss $\frac{d E}{E d x}[\% / \mathrm{m}]$ & 0.12 & 0.0088 & 9.57 & 0.73 \\
Multiple scattering (projected root mean & 73.5 & 4.72 & 73.5 & 4.72 \\
$\quad$ square (RMS) angle) $\left[\mu \mathrm{rad} / \mathrm{m}^{\frac{1}{2}}\right]$ & & & 20 & 312 \\
Electron capture length $[\mathrm{cm}]$ & $\ldots$ & $\ldots$ & 0.028 & 0.018 \\
Electron stripping length $[\mathrm{cm}]$ & $\ldots$ & $\ldots$ & 24.5 & 0.63 \\
ECPP interaction length $[\mathrm{cm}]$ & $\ldots$ & $\ldots$ & 2.5 & 2.2 \\
Nuclear interaction length (inc. fragmentation) $[\mathrm{cm}]$ & 38.1 & 38.1 & 33.0 & 19.0 \\
EMD length [cm] & $\ldots$ & $\ldots$ & \\
\hline \hline
\end{tabular}

ion-specific beam losses [1]. Ions undergo nuclear fragmentation and electromagnetic dissociation (EMD). An impinging nucleus may lose one or several nucleons, in particular neutrons, through EMD. In an EMD process, one of the lead ions $\left({ }^{208} \mathrm{~Pb}^{82+}\right)$ makes a transition to an excited nuclear state that then decays with the emission of a neutron, leaving a lighter isotope of lead $\left({ }^{207} \mathrm{~Pb}^{82+}\right)$. Another important EMD process is the production of a secondary beam of ${ }^{206} \mathrm{~Pb}^{82+}$. However, the rates of transmutation of the lead ions to isotopes of lighter elements are very small compared with the processes in which the lead ion emits one or two neutrons. Moreover, the nuclei may also split up in smaller fragments through nuclear inelastic reactions.

Once the ions have fragmented, the resulting hadronic shower behaves similarly for both particle types (protons and heavy ions) and the heat deposition is proportional to the beam energies [1]. Other important physical processes that occur when both heavy nuclei and protons traverse the collimator material are the energy loss through ionization and the change of direction through many small-angle scattering events, known as multiple Coulomb scattering (MCS). However, the ionization energy loss is much higher for ions than for protons. The energy loss through ionization, which is described by the well-known Bethe-Bloch formula [21], rises proportionally with the square of the particle's atomic number, which means that a lead ion will lose more energy per unit path length in a material than, for instance, a proton. Consequently, this means that the energy deposition from a lead ion will be much more concentrated. Angular deviation can also be caused by nuclear elastic scattering, which is a significant effect for protons but negligible for ${ }^{208} \mathrm{~Pb}^{82+}$.

The role of TCPs in the LHC collimation system is to intercept halo and off-momentum particles whilst increasing their betatron amplitude by means of MCS. In the case of protons, it can be assumed that the main action of TCPs on an intercepted particle is scattering while energy loss has a comparably small effect on the particle trajectories. This assumption is however no longer valid for heavy ions because:
(1) The relative energy loss due to ionization is two orders of magnitude larger for ions than for protons.

(2) Peripheral collisions of heavy ions with collimator nuclei lead to nucleon losses by hadronic fragmentation and EMD, whose effects are comparable to a change of longitudinal particle momentum from a beam dynamics point of view.

(3) The root mean square (RMS) scattering angle is proportional to the square root of the penetration depth and the proportionality coefficient is similar for protons and ions.

As a conclusion, due to the complexity of the physical processes involved, the collimation system tends to put ions on trajectories characterized by large momentum errors but of moderate betatron amplitude increase when compared to protons. This is because nuclear fragmentation and EMD in the TCPs create fragments with a wide range of Z/A (i.e. atomic number/atomic mass) ratios. In addition, TCSs are designed to cut into the betatron amplitude of secondary halo particles scattered out of the TCPs. Thus, since ion beam fragments scattered out of the TCPs exhibit only a small betatron amplitude increase with respect to primary particles, such fragments have a high probability of exiting the TCP without being intercepted by the TCS. These fragments can consequently be lost where the dispersion has grown sufficiently large in the LHC machine. As a result, the cleaning efficiency for ${ }^{208} \mathrm{~Pb}^{82+}$ ion beams in the LHC is substantially smaller than for protons. The LHC collimation setup, designed as a three-stage system for proton operation, is thus effectively reduced to a one-stage system for ions [1].

\section{BEAM IMPACT ON A TERTIARY COLLIMATOR}

\section{A. Tertiary collimator design}

Like most collimators, the TCT consists of two parallel jaws contained in a vacuum tank, with the beam passing through the center of the jaw gap (Fig. 1). Being in proximity to the beam, the collimator jaws are continuously exposed to 


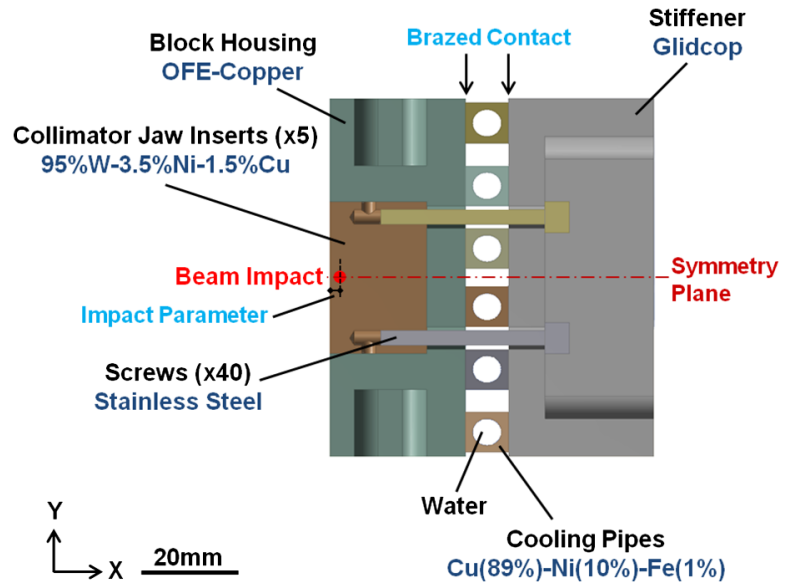

FIG. 2. Detailed cross-sectional view of the $x-y$ plane of a horizontal TCT right jaw assembly. The blue labeling gives the material of each respective component while the red dot indicates the location of the beam impact with a certain impact parameter in case of an accident scenario.

direct interaction with high-energy particles. For optimal performance, the jaws are centered around the actual orbit through a beam-based alignment procedure [22]. Each TCT jaw has a total length of $1.2 \mathrm{~m}(1 \mathrm{~m}$ active length $+0.1 \mathrm{~m}$ tapering at the upstream and downstream parts of the jaw), and consists of five inserts made of a tungsten heavy alloy, known commercially as INERMET ${ }^{\circledR} 180$. These five blocks are then placed into a copper housing and fixed with stainless steel screws to the jaw assembly. The water cooling pipes are an integral part of the collimator structure, with a total flow rate of $25 \mathrm{l} / \mathrm{min}$. Two stepping motors per jaw allow independent adjustment (with an accuracy of $5 \mu \mathrm{m}$ ) of jaw tilt and jaw position relative to the beam center. A detailed view of the jaw assembly is shown in Fig. 2.

\section{B. Studied accident scenario}

One of the serious accident scenarios identified within the LHC is an asynchronous beam dump [23]. An asynchronous beam dump scenario refers to a spontaneous misfiring of one (or more) of the 15 extraction kicker magnets (MKDs) that causes a trigger of all the other beam dump kickers outside the particle-free abort gap. The result of this accident scenario is that some bunches of the beam experience a kick during the kicker field ramp, which is smaller than the nominal kick, and consequently, they circulate for 1 turn before being kicked out.

Unsynchronized aborts cannot be excluded and they can seriously damage downstream accelerator components, in particular the septum magnets (MSDs), the TCTs and the experimental low- $\beta$ triplet magnets. However, a system of protection devices, comprising dedicated fixed (TCDS) and movable (TCDQ) dilution devices, are placed in front of the MSD and the Q4 quadrupole (Fig. 3) in order to prevent the misdirected bunches from causing local equipment damage. If the collimators are set up correctly, sensitive equipment, such as the SC magnets, are in the shadow of the TCDQ (dump protection collimator) and are thus well protected from the misdirected bunches in case of such a failure.

The probability that an asynchronous beam dump event happens was originally estimated to be once per year [23]. However, the probability that a collimator is hit directly by a full intensity bunch is lower since it comes as a result of other concurrent errors such as collimator settings and orbit errors. In this context, a scenario with such combined errors [25] was considered in this paper as a conservative case study in order to understand the consequences of a potentially severe beam impact event on a tungsten-based collimator.

This paper presents case studies based on this worst case error scenario. General inputs have been used in a simplified impact model to investigate the damage extent caused by proton and lead ion beams in case of direct beam impact on a TCT. Compared with the nominal proton beams, the relatively low intensities and larger bunch spacing of the lead ion beams result in an even smaller

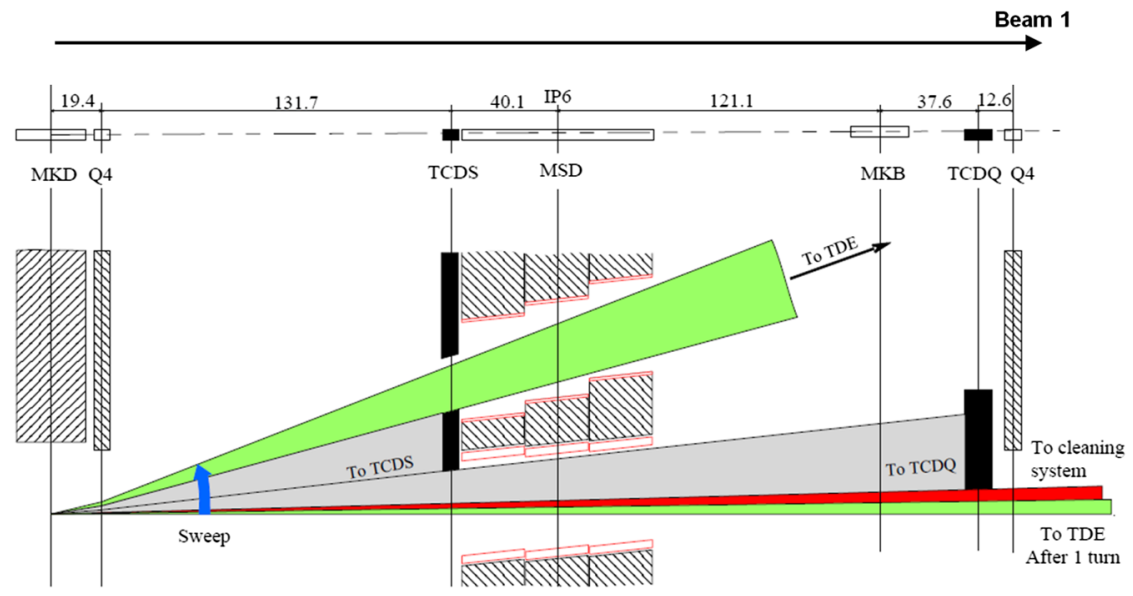

FIG. 3. Schematic and functional layout of the TCDS and the TCDQ diluter elements for Beam 1 (adapted from [24]). 
probability of such a scenario. In the case studies presented in this paper, the bunch has a transverse Gaussian profile of $0.3 \mathrm{~mm}\left(\sigma_{x}\right) \times 0.3 \mathrm{~mm}\left(\sigma_{y}\right)$ RMS beam size. As specified in Table I, the nominal ion and proton bunch intensities are equivalent to $7 \times 10^{7}$ ions and $1.15 \times 10^{11}$ protons, respectively. Moreover, the impact parameter, which is the transverse depth at which the beam first makes contact with the jaw material, is assumed to be $0.5 \mathrm{~mm}$ (Fig. 2) in order to investigate the effects of grazing of the misdirected bunch on the TCT's planar collimating surface.

\section{NUMERICAL ANALYSIS}

\section{A. Simulation tools}

The interaction of high-energy particle beams with matter during a beam impact induces various fast and complex thermomechanical phenomena. This effect, together with the complexity of the collimator structure, make the implementation of a numerical FEM approach, as opposed to analytical solutions, highly necessary to properly study such complex phenomena [26].

The first step in conducting such a numerical approach is to obtain detailed energy deposition maps that are then used as an input for the thermomechanical calculations of the collimator structure. For this study, the evaluation of the thermal loads on the hit material was done using the Monte Carlo based statistical code FLUKA [27,28]. FLUKA models of the jaw inserts were set up and full shower simulations provided energy deposition distributions (in $\mathrm{GeV} / \mathrm{cm}^{3} /$ proton) for the defined accident cases. The calculation was performed considering a large number of primary particles ( 200,000 in these case studies), thus ensuring that statistical errors, related to the adopted scoring mesh, were kept to a minimum. The results were then normalized to one ideal proton, allowing the FLUKA maps to be rescaled by the real bunch intensity. The FLUKA 3D maps, giving the spatial distribution of the specific energy deposited on the jaw inserts, are shown in Fig. 4. These energy deposition maps were then loaded in the FEM 3D model through dedicated subroutines in order to provide the input thermal load in terms of power density distribution (in $\mathrm{W} / \mathrm{m}^{3}$ ) for the FEM solution.

Nonlinear, transient thermal and structural analyses were then performed sequentially using the finite element code ANSYS $^{\circledR}$ [29] in order to evaluate the thermally induced effects provoked by the beam impact on the TCT. Such effects include the sudden nonuniform temperature increase on the jaw inserts that, in turn, gives rise to thermal stresses and deformations.

\section{B. Finite element modeling}

\section{Component setup and finite element discretization}

A detailed 3D model of the TCT collimator jaw assembly (Fig. 5) was imported into the finite element program ANSYS $^{\circledR}$ in order to simulate the collimator structure.
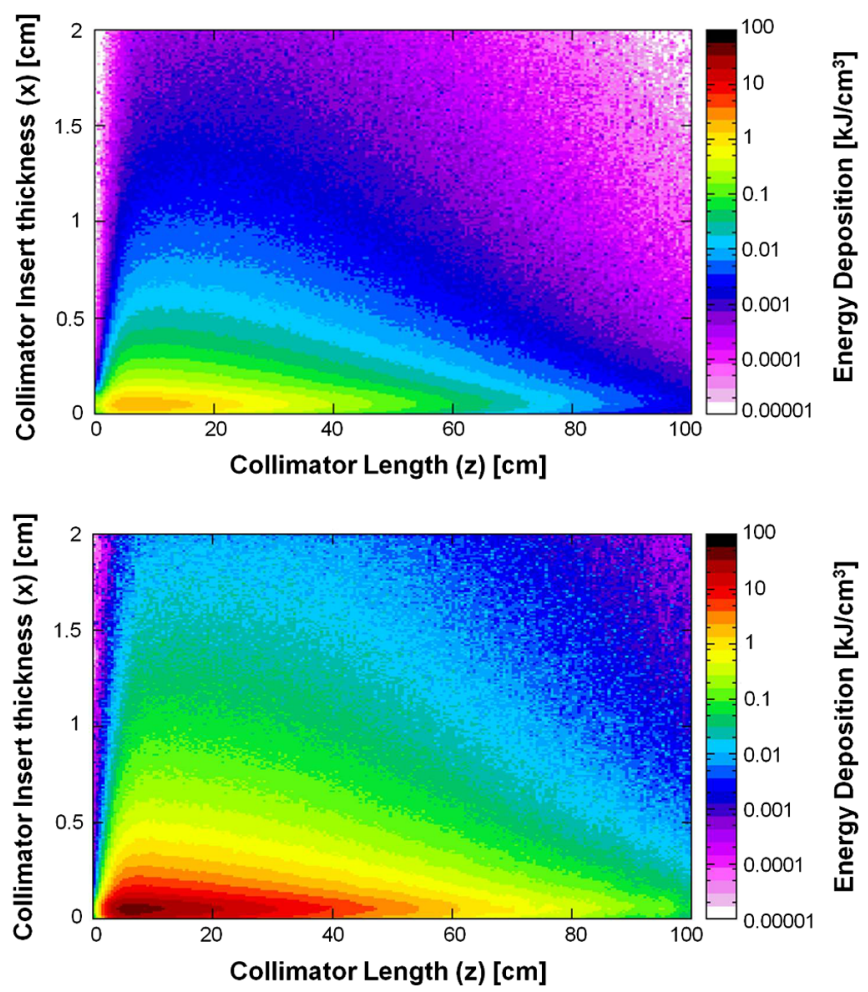

FIG. 4. Energy deposition cuts in the $\mathrm{x}-\mathrm{z}$ plane at the collimator insert half-height (symmetry plane). 1 nominal bunch of lead ions $\left(7 \times 10^{7}\right.$ ions, $\left.2759 \mathrm{GeV} / \mathrm{n}\right)$ (upper). 1 nominal bunch of protons $\left(1.15 \times 10^{11}\right.$ protons, $\left.7 \mathrm{TeV}\right)$ (lower).

The considered beam impact leads to a symmetrical energy deposition in the longitudinal (x-z) plane of the collimator jaw. Thus, given that the model proved to be symmetric with regard to geometry, loads, boundary conditions and material properties about the mid-plane of the jaw inserts, it was only necessary to model the lower or the upper half of the collimator jaw structure. These symmetry conditions reduced the computational time necessary to perform the calculation without compromising accuracy. The sequential thermal and structural FEM simulations were performed on the model of the lower symmetrical half of the right jaw assembly shown in Fig. 6, taking also into account all contact interfaces between the various components.

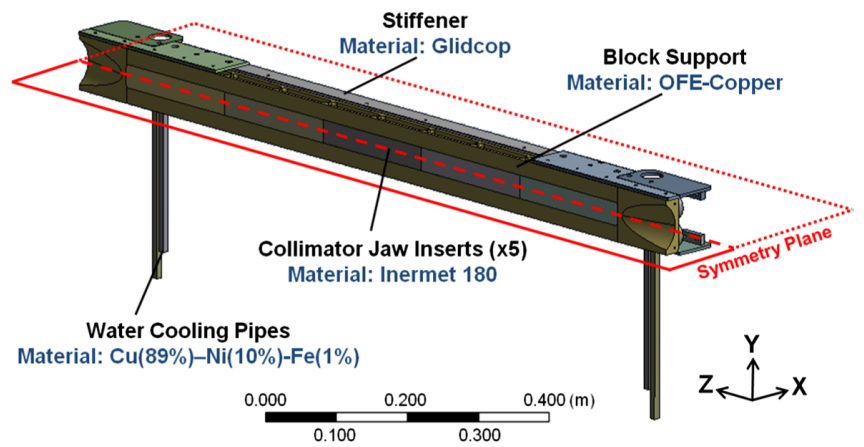

FIG. 5. 3D model of the right jaw assembly of a TCT. The symmetry plane ( $\mathrm{x}-\mathrm{z}$ plane) is shown in red. 
Symmetry Plane

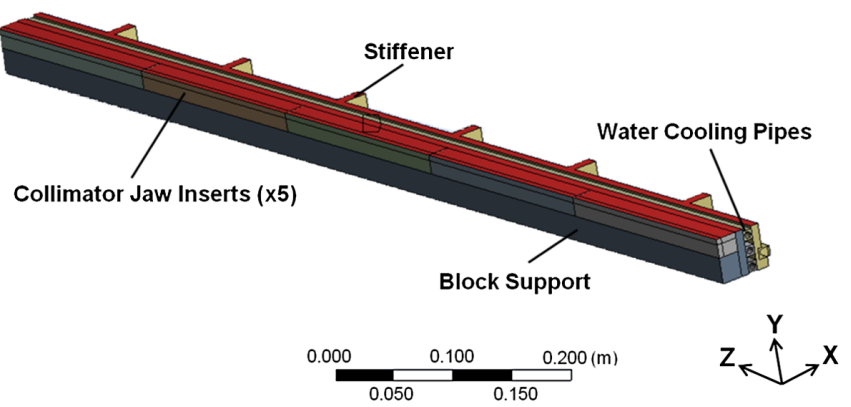

FIG. 6. 3D model of the lower symmetrical half of the right jaw assembly of a TCT. The symmetry plane (x-z plane) is shown in red.

A mesh convergence sensitivity study is required to find a satisfactory balance between accuracy and computational resources. Such a study identifies the mesh density that is sufficient to capture the energy deposited on the collimator jaw inserts. FLUKA simulations were carried out for different mesh densities of a particular case to see, at what stage, the energy deposition values converge. From these studies, it was concluded that, for the thermal analyses, a finest mesh size of $0.1 \mathrm{~mm}(\mathrm{x}) \times 0.1 \mathrm{~mm}(\mathrm{y}) \times 5 \mathrm{~mm}(\mathrm{z})$, implemented within a region of $5 \mathrm{~mm}(\mathrm{x}) \times 5 \mathrm{~mm}(\mathrm{y})$ around the beam impact location in the transverse plane, would be sufficient to correctly enclose the location of the maximum energy deposition (Fig. 7). The remaining collimator components, such as the block housing, the water pipes and the back stiffener, were then discretized with a coarser mesh due to computational requirements. Furthermore, different meshes were employed for the thermal and structural analyses (Fig. 7). Thus, the temperature distribution as a function of time, obtained from the thermal analyses, was interpolated and applied as a load at different time steps in the structural calculations for the evaluation of thermally induced stresses.
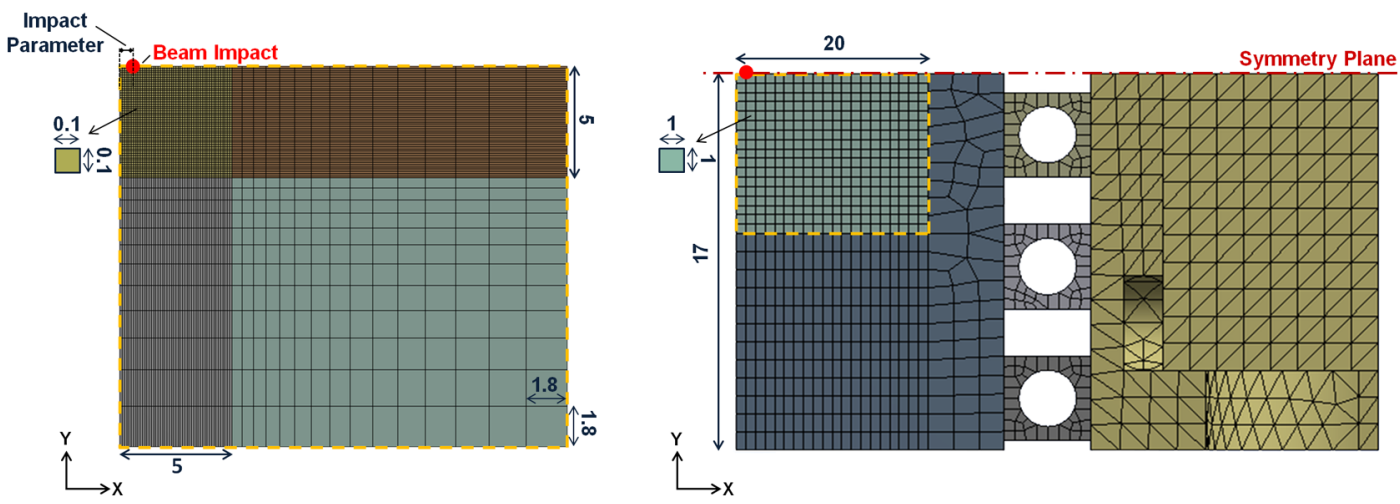

FIG. 7. Finite element discretization of the collimator jaw assembly. A detailed cross-sectional (x-y) view of the collimator jaw inserts as discretized for the thermal analyses (left). A cross-sectional view of the discretized model of the collimator jaw assembly as used for the structural analyses (right). The only difference between the thermal and structural meshes is the mesh of the collimator jaw inserts (enclosed within the dashed line), with a coarser mesh used for the structural analyses. All dimensions are in mm. The total number of elements used for the thermal and structural analyses are 1,056,265 and 368,335, respectively.
Material modeling is a very important step in any finite element analysis as it essentially drives the behavior of the model and thus influences the results. In cases of shock beam impact on a structure, as presented in this paper, a large temperature variation is experienced. Thus, temperature-dependent material properties are deemed necessary in order to correctly evaluate the thermomechanical response of the TCT structure.

When a high-energy beam of particles impacts the collimator structure during an asynchronous beam dump, a great percentage of the energy is deposited within the collimator jaw inserts, thus making the material modeling of the latter extremely critical. As explained in Sec. III A, the jaw inserts of LHC TCTs are made of INERMET ${ }^{\circledR} 180$, which is the commercial name for a tungsten heavy metal alloy with a composition of $95 \mathrm{wt} \% \mathrm{~W}-3.5 \mathrm{wt} \% \mathrm{Ni}-1.5 \mathrm{wt} \%$ $\mathrm{Cu}$. Tungsten is the main component of the alloy (present in $\sim 95 \mathrm{wt} \%)$ and it is the reason for its high density $\left(\sim 18,000 \mathrm{~kg} / \mathrm{m}^{3}\right)$. Nickel and copper serve as a binder matrix that holds the brittle tungsten grains together while also making the alloy ductile and easy to machine. A typical microstructure (Fig. 8) consists of spherical tungsten grains (20 to $60 \mu \mathrm{m}$ in diameter) that are embedded in a tough metallic matrix. While the grains are nearly pure bodycentered-cubic tungsten, the face-centered-cubic binder matrix contains $\sim 20 \mathrm{wt} \%$ tungsten in solid solution [30].

Research in tungsten heavy alloys is mostly boosted by ballistic applications of such alloys as anti-armor kinetic energy penetrators. Thus, data providing properties of such materials under extreme conditions is very scarce. Since material properties of INERMET ${ }^{\circledR} 180$ at high temperatures were not easily available, material characterization tests of this alloy have been commissioned at different laboratories in order to obtain a full thermal and structural characterization of this alloy under varying temperature and strain-rate conditions. 


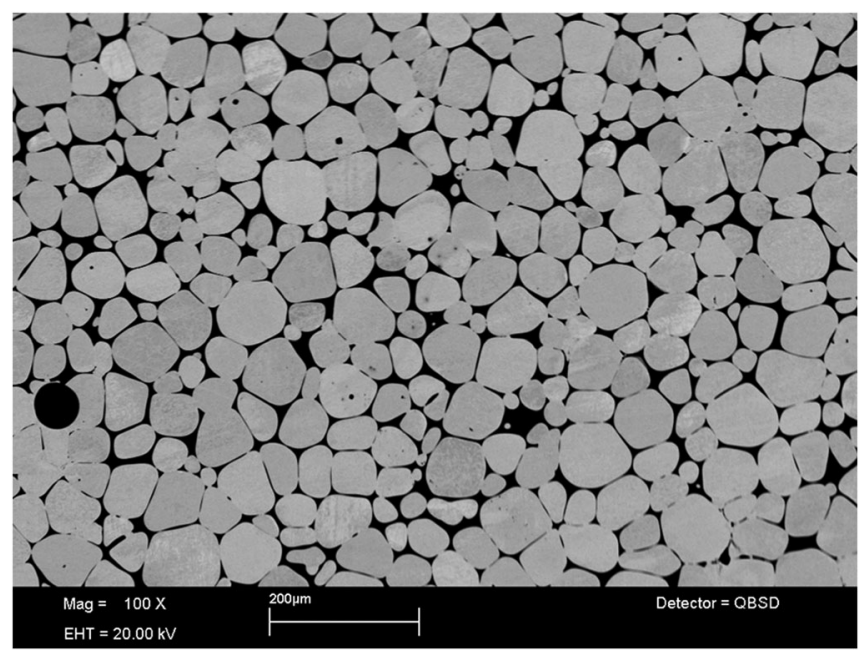

FIG. 8. Scanning electron microscope (SEM) image at low magnification $(100 \times)$ showing the typical microstructure of INERMET $^{\circledR} 180$. The tungsten grains are clear while the binder phase appears black.

A thermophysical analysis of INERMET ${ }^{\circledR} 180$ was performed within the Energy Department of the Austrian Institute of Technology [31]. This test campaign involved the measurements of thermal conductivity, thermal diffusivity, specific heat capacity, and linear thermal expansion of INERMET $^{\circledR} 180$ between room temperature up to $1450^{\circ} \mathrm{C}$ (Fig. 9). Moreover, the structural characterization of INERMET $^{\circledR} 180$ was carried in collaboration with DYNLab at the Politecnico di Torino. A series of tests at different temperatures and strain-rates were performed in order to obtain information about the thermal softening and strain-rate behavior of the material. The specimen was heated using an induction coil system. This system was controlled with feedback on the temperature measurement


FIG. 9. Thermophysical properties of INERMET ${ }^{\circledR} 180$ measured between room temperature up to $1450{ }^{\circ} \mathrm{C}$ at the Austrian Institute of Technology.

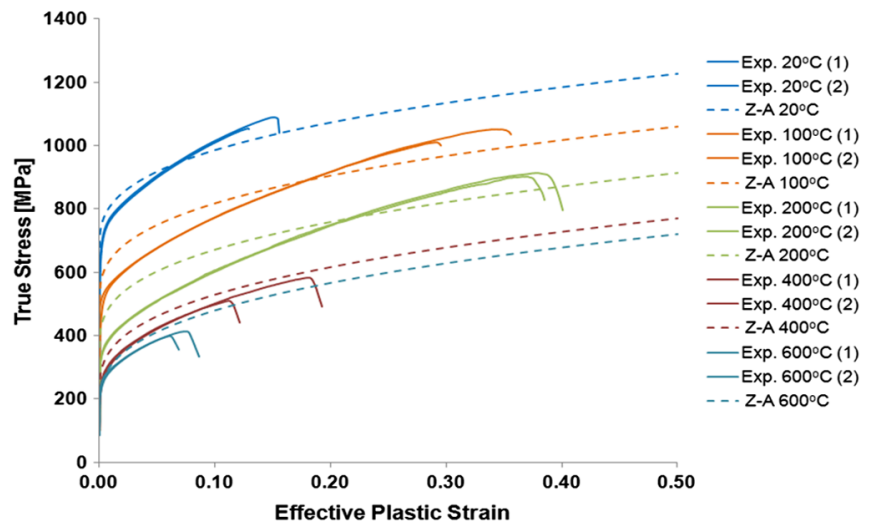

FIG. 10. Mechanical properties of INERMET ${ }^{\circledR} 180$ measured between room temperature up to $600{ }^{\circ} \mathrm{C}$ at the Politecnico di Torino. Two sets of experimental data (obtained from two samples in the same test conditions) are shown for each temperature at a fixed strain rate of $10^{-3} \mathrm{~s}^{-1}$. The Z-A model was then used to obtain a unique strength model for the material with a sufficient level of accuracy.

obtained using thermocouples directly welded on the specimen surface. The experimental data was processed via a numerical inverse method based on FEM numerical simulations [32] and the best fit of the experimental data was obtained with a modified version of the ZerilliArmstrong (Z-A) model [33] as given in [34] (Fig. 10).

\section{Loading and boundary conditions}

An asynchronous beam dump entails a rapid energy deposition on the collimator jaw assembly in case of a beam impact. Such a thermal load was applied on the collimator as an internal heat generation through the application of body loads on the ANSYS ${ }^{\circledR}$ 3D model. Transient thermal analyses were performed for the whole collimator structure, with thermal boundary conditions taking into account both the energy rate $\left[\mathrm{W} / \mathrm{m}^{3}\right]$ deposited on the jaw as well as the heat convection on the inner wet surface of the cooling pipes. Moreover, the collimator jaw assembly was simply supported at its extremities. The effect of the inner pressure caused by the water flow on the cooling pipes was also considered and a load equivalent to a pressure of 15 bar [35] was applied to the inner surface of the cooling pipes in the finite element model.

For the impact scenario under study, the thermal shock duration, $\tau_{\text {shock }}$, is equal to the length of one bunch $(1 \mathrm{~ns})$. As shown by Kalbreier et al. [36], it is possible to assume that no heat diffusion occurs during $\tau_{\text {shock }}$ because the characteristic thermal diffusion time, $\tau_{\text {diffusion, is much }}$ longer than $\tau_{\text {shock}}$. Using Eqs. (1) and (2) to calculate $\tau_{\text {diffusion }}$ for the jaw inserts (material: INERMET ${ }^{\circledR} 180$ ), it was found to be $\sim 306 \mu$ s, considering the transverse edge length of one mesh element in the thermal solution $(0.1 \mathrm{~mm})$ as the typical dimension of the structure across which heat diffusion is simulated. Eqs. (1) and (2) are given by: 


$$
\begin{gathered}
\tau_{\text {diffusion }}=\frac{l^{2}}{\kappa_{\mathrm{cc}}}, \\
\kappa_{\mathrm{cc}}=\frac{k}{\rho c_{\mathrm{p}}},
\end{gathered}
$$

where $l$ is the typical dimension along which heat diffusion is simulated, $\kappa_{\mathrm{cc}}$ is the thermal diffusivity, $k$ is the thermal conductivity, $\rho$ is the density, and $c_{\mathrm{p}}$ is the specific heat capacity at constant pressure.

Comparing the rapidity of the beam impact duration $(1 \mathrm{~ns})$ with the typical thermal diffusion time $(\sim 306 \mu \mathrm{s})$, it can be concluded that heat conduction plays a minor role on the short timescale. Moreover, in this case, thermal deformations are too small to affect the overall structural response. Thus, this case can be considered as a weaklycoupled thermoelastic problem, making it possible to decouple and sequentially solve the transient thermal and structural analyses. This adopted approach compromises the computational time necessary to perform the analyses because very short time steps must be used to maintain the validity of the weakly-coupled thermoelastic approach. The choice of the used time steps will be explained later by Eq. (4).

The thermostructural behavior of the collimator jaw assembly is correctly captured through the choice of appropriate integration time steps (ITSs) and a good mesh size. The rapid temperature increase provokes a dynamic response of the structure in terms of longitudinal and flexural vibrations, as well as propagation of thermal stress waves. The frequency range of these phenomena starts from $\sim 105 \mathrm{~Hz}$, which corresponds to the first period of flexural oscillation of the jaw assembly, as calculated by Eq. (3):

$$
t_{\text {flex }}=\frac{2}{\pi} \sqrt{\frac{M L^{3}}{E I}}
$$

where $M, L$, and $I$ are respectively the mass, length and cross-sectional inertia of the collimator jaw assembly, and $E$ is an equivalent Young's Modulus obtained as an average value between the various materials of the jaw assembly.

The ITS must be optimized depending on the mesh size. This is given by Eq. (4) that represents the Courant criterion [37] for the solution of dynamic structural problems:

$$
\Delta t \leq \frac{0.9 L_{\text {mesh }}}{c}
$$

where $L_{\text {mesh }}$ is a typical mesh size in the structural solution and $c$ is the speed of sound in INERMET ${ }^{\circledR} 180$.

The transient thermal and structural load cases were implemented as a sequence of load steps as shown in Table III. The minimum step size was fixed to $0.1 \mu$ s for the structural analyses. This was based on a preliminary analytical estimation performed using Eq. (4) with $L_{\text {mesh }}=$ $1 \times 10^{-3} \mathrm{~m}$ and $c \approx 5180 \mathrm{~m} / \mathrm{s}$. Temperature distributions from the thermal analyses were applied at different time steps in the structural analyses (Table III). ANSYS ${ }^{\circledR}$ linearly interpolates between load time steps, therefore closely following the actual temperature evolution. Moreover, as can be seen in Table III, the ITS was progressively incremented to avoid excessive CPU time of calculation while still ensuring that the higher frequency phenomena are correctly captured on the very short timescale.

\section{RESULTS AND EVALUATION}

\section{A. Thermal analyses}

An initial comparison between protons and lead ions can be done by evaluating the energy deposited by 1 nominal bunch of each particle type on the collimator jaw inserts. It is observed from Fig. 4 that the energy deposited by a nominal ion bunch is around two orders of magnitude smaller than that for a nominal proton bunch. This results

TABLE III. Load step sequences used for the FEM thermal and structural analyses. The first load step represents the beam impact. The computational time needed to achieve complete transient thermal and structural solutions was $\sim 1$ day and $\sim 4$ weeks per solution respectively on a $32 \mathrm{~GB}$ RAM 4-core machine with a processor speed of $4.0 \mathrm{GHz}$.

\begin{tabular}{lcccc}
\hline \hline $\begin{array}{l}\text { Load step } \\
\text { number }\end{array}$ & $\begin{array}{c}\text { Time at end } \\
\text { of loadstep [s] }\end{array}$ & $\begin{array}{c}\text { Thermal ITS } \\
\Delta t_{\text {thermal }}[\mathrm{s}]\end{array}$ & $\begin{array}{c}\text { Structural ITS } \\
\Delta t_{\text {structural }}[\mathrm{s}]\end{array}$ & $\begin{array}{c}\text { Temperature Load } \\
\text { Time Step [s] }\end{array}$ \\
\hline 1 & $1.00 \times 10^{-9}$ & $1.25 \times 10^{-10}$ & $1.25 \times 10^{-10}$ & $1.00 \times 10^{-9}$ \\
2 & $1.00 \times 10^{-6}$ & $1.00 \times 10^{-7}$ & $1.00 \times 10^{-7}$ & $1.00 \times 10^{-6}$ \\
3 & $1.00 \times 10^{-4}$ & $2.50 \times 10^{-6}$ & $1.00 \times 10^{-6}$ & $1.00 \times 10^{-4}$ \\
4 & $2.00 \times 10^{-3}$ & $2.00 \times 10^{-5}$ & $1.00 \times 10^{-5}$ & $2.00 \times 10^{-3}$ \\
5 & 0.02 & $2.00 \times 10^{-4}$ & $1.00 \times 10^{-4}$ & $5.00 \times 10^{-3}, 0.01,0.02$ \\
6 & 0.06 & $5.00 \times 10^{-4}$ & $2.50 \times 10^{-4}$ & $0.04,0.06$ \\
7 & 0.12 & $1.00 \times 10^{-3}$ & $2.50 \times 10^{-4}$ & 0.12 \\
8 & 0.25 & $5.00 \times 10^{-3}$ & $1.25 \times 10^{-3}$ & 0.25 \\
9 & 1.00 & 0.05 & $2.50 \times 10^{-3}$ & 1.00 \\
10 & 5.00 & 0.20 & $5.00 \times 10^{-3}$ & 5.00 \\
\hline \hline
\end{tabular}


from the fact that the stored energy of the nominal ion beam is only $1 \%$ of that of the nominal proton beam (Table I).

Given the energy rate map from FLUKA, transient thermal analyses were then performed with $\mathrm{ANSYS}^{\circledR}$ to calculate the temperature distribution and its evolution over time. The collimator jaw receives all the energy during the $1 \mathrm{~ns}$ beam impact duration, with the maximum temperature $\left(T_{\max }\right)$ reached on the jaw inserts. The temperature computation was done starting from the internal energy value with the heat capacity and density of the solid material. For this reason, the simulated temperature values are realistic only in the solid material part of the component. A different $T_{\max }$ is reached for the nominal proton and ion beam impacts (Figs. 11 and 12). This subsequently influences the dimension of the molten region on the jaw inserts (if any) and the extent of the beam-induced damage.

Another interesting aspect was to simulate the number of protons per bunch that would give the same $T_{\max }$ as 1 nominal ion bunch, considering the same beam size and the same impact parameter. The graph in Fig. 13 shows that $4.48 \times 10^{9}$ protons per bunch would in fact approximately give the same $T_{\max }$ as 1 nominal ion bunch. However, some differences between the two temperature profiles can be observed with the main observable difference being the discrepancy between the energy deposition values close to the jaw entrance. This difference is caused by ionization, confirming that the relative energy loss due to ionization is two orders of magnitude larger for heavy ions than for protons, as given in Table II.

From the thermal analyses, it can be concluded that the heat deposition, and consequently the damage caused by the impact of a nominal ion bunch, nowhere exceeds that

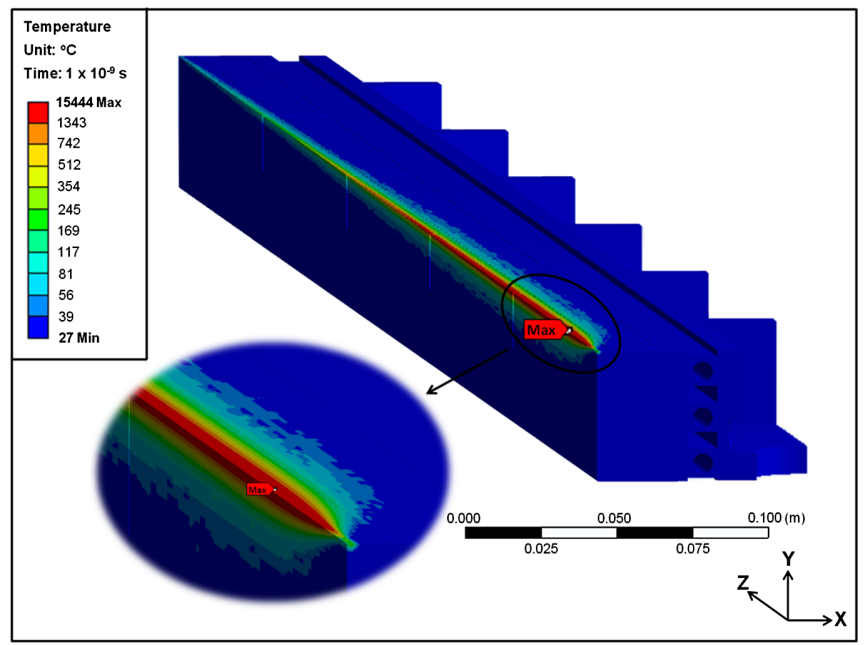

FIG. 11. Temperature distribution provoked by the nominal proton beam impact at $1 \mathrm{~ns}$. The red region represents temperatures that exceed the melting temperature of INERMET ${ }^{\circledR} 180$ $\left(\sim 1343^{\circ} \mathrm{C}\right)$ and is thus an indication of the extent of the groove formation on the surface of the collimator jaw inserts. A detailed view is shown in the inset.

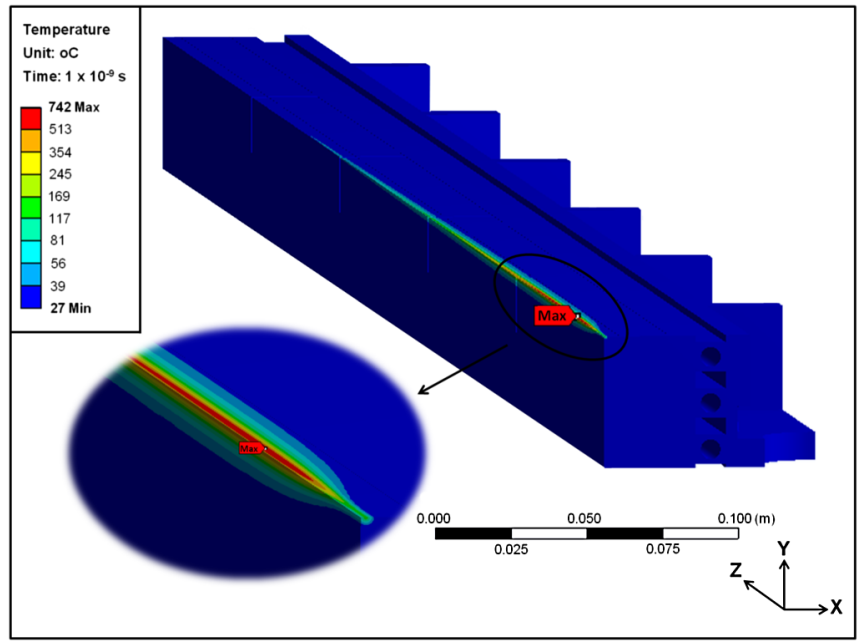

FIG. 12. Temperature distribution provoked by the nominal lead ion beam impact at $1 \mathrm{~ns}$. The melting temperature of INERMET $^{\circledR} 180\left(\sim 1343^{\circ} \mathrm{C}\right)$ is not exceeded in any region in this case. A detailed view is shown in the inset.

resulting from a nominal proton bunch. Thus, in order to be able to qualitatively compare the effects resulting from the differences between the interactions of protons and heavy ions with matter, it was decided to consider impact cases of the two particle types where the same $T_{\max }$ is reached. In fact, some differences in the temperature profiles of these two cases were observed, with such discrepancies mainly arising from ionization effects (Fig. 13). Section V B will now investigate and discuss if such differences will play any role in the structural behavior of the collimator when subject to an asynchronous beam dump.

\section{B. Structural analyses}

Rapid energy deposition during a beam accident scenario results in a fast temperature increase within the hit structure. Thermal expansion is prevented by the inertia

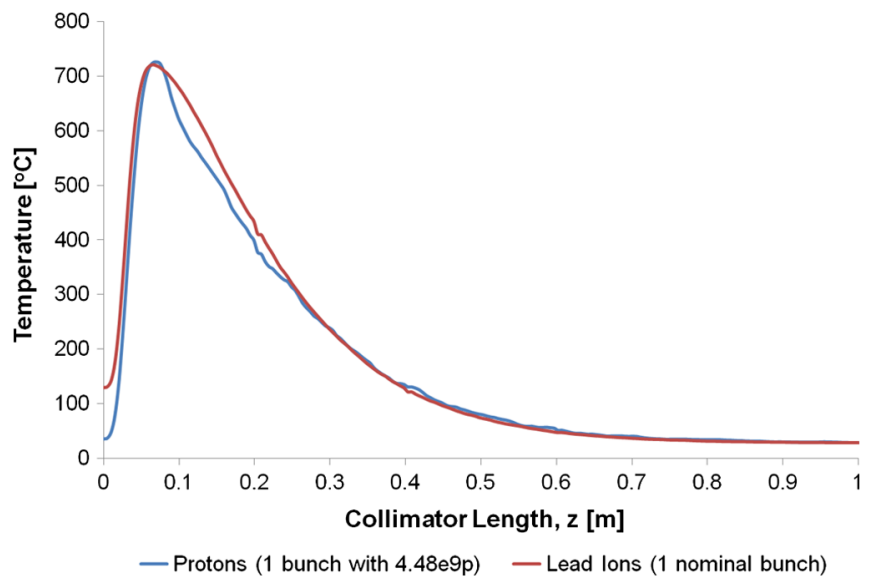

FIG. 13. Temperature peak profiles within the jaw inserts along the beam direction for heavy ions and protons. 
of the body and consequently, dynamic structural response takes place. The adopted numerical FEM approach evaluates the collimator structure in the elastic-plastic domain of its constituent materials, allowing also for the study of potential permanent damage provoked by the thermal shock.

Once the expected temperature increase is known, the range of compressive stresses and the plasticization provoked by the thermal shock can be estimated. Assuming that no longitudinal expansion occurs, compressive strains, $\epsilon$, and linear elastic stresses, $\sigma^{\text {linear }}$, can be evaluated using Eqs. (5) and (6), respectively:

$$
\begin{gathered}
\epsilon_{z_{\max }}=-\alpha \Delta T_{\max }, \\
\sigma_{z_{\max }}^{\text {linear }}=-\frac{E \alpha \Delta T_{\max }}{1-2 \nu},
\end{gathered}
$$

where $E$ and $\alpha$ are the Young's Modulus and the coefficient of thermal expansion of INERMET ${ }^{\circledR} 180$, respectively, $\Delta T_{\max }=T_{\max }-T_{\text {ref }}, \quad T_{\text {ref }}$ is $27^{\circ} \mathrm{C}$ and $\nu$ is the Poisson's ratio.

Given that $\Delta T_{\max }$ and the jaw insert material are set to be the same for the two considered cases, it is expected that the values of $\epsilon_{\mathrm{z}_{\max }}$ and $\sigma_{\mathrm{z}_{\max }}^{\text {linear }}$ would also be the same. Thus, considering the ion beam impact case, Fig. 14 shows a contour plot of the longitudinal elastic strain on the collimator jaw assembly following the impact of 1 nominal ion bunch. Good agreement is achieved for the normal elastic strain in the ion beam impact case on comparing the numerical values shown in Fig. 14 and the analytical values calculated with Eq. (5) using $\alpha=5.25 \times 10^{-6} \mathrm{~K}^{-1}$ and $T_{\max }$ as given in Fig. 12. The behavior of the compressive stress developed within the collimator jaw assembly, both for the ion and proton beam impacts, is very similar to that

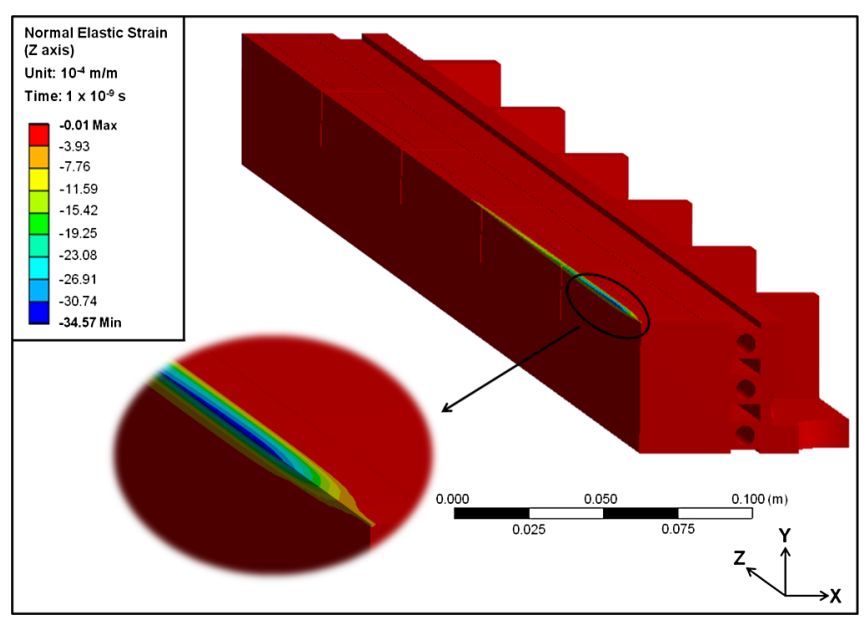

FIG. 14. Normal elastic strain along the z-direction on the collimator jaw assembly after the nominal ion beam impact. A detailed view is shown in the inset. A similar plot with comparable peak values has been achieved for the case of the proton beam impact $\left(4.48 \times 10^{9}\right.$ protons $)$. of the normal elastic strain shown in Fig. 14, with a maximum compressive longitudinal stress of $\sim 3 \mathrm{GPa}$ developed at the same location as the maximum compressive longitudinal strain. This value of maximum compressive stress is also comparable with that achieved with Eq. (6), using $E=360 \mathrm{GPa}$ and $\nu=0.28$ in addition to $\alpha$ and $T_{\max }$ as given previously.

The numerical results of the dynamic response of the collimator jaw assembly, as calculated with $\mathrm{ANSYS}^{\circledR}$, will now be presented. The effect of the temperature distributions shown in Figs. 11 and 12 can be considered as two dynamic thermal loads: axial force and bending moment. Such loads help to explain the longitudinal and flexural vibrations, as well as the dynamic thermal stresses that are obtained from the FEM analysis. It can be observed from Fig. 15 that the jaw assembly, hit by the high-energy particle beam, shows a dynamic flexural response with a main frequency of $\sim 100 \mathrm{~Hz}$. The main frequency of flexural oscillation, as analytically calculated by Eq. (3), is thus correctly predicted by numerical simulations. Moreover, it can be noticed that the jaw assembly vibrates, with an underdamped response, around its quasistatic deflected position. Theoretically, for a given system, the value of the quasistatic deflection should be equal to the value of the dynamic deflection at the end of the dynamic response. However, it is calculated that for both the proton and ion case studies, there is a discrepancy of $\sim 3.65 \%$ between these two values. Given the complexity of these FEM simulations, this is still considered as being within an acceptable limit. The simulated dynamic thermal stresses are also given in Fig. 16.

An important mode of failure during high velocity impact is spallation. Spallation is the process of internal failure or

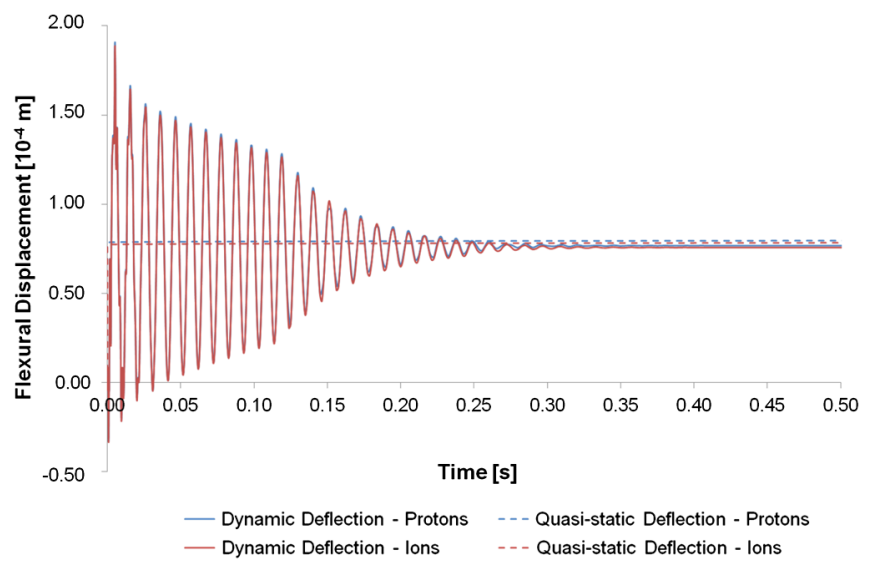

FIG. 15. Dynamic flexural displacement (x-direction) of the collimator jaw assembly as provoked by the proton ( 1 bunch with $4.48 \times 10^{9}$ protons) beam impact, and as provoked by the ion (1 nominal ion bunch) beam impact. The quasistatic deflection due to the thermal bending moment is also shown for both cases. No significant difference can be observed between the proton and ion case studies. 


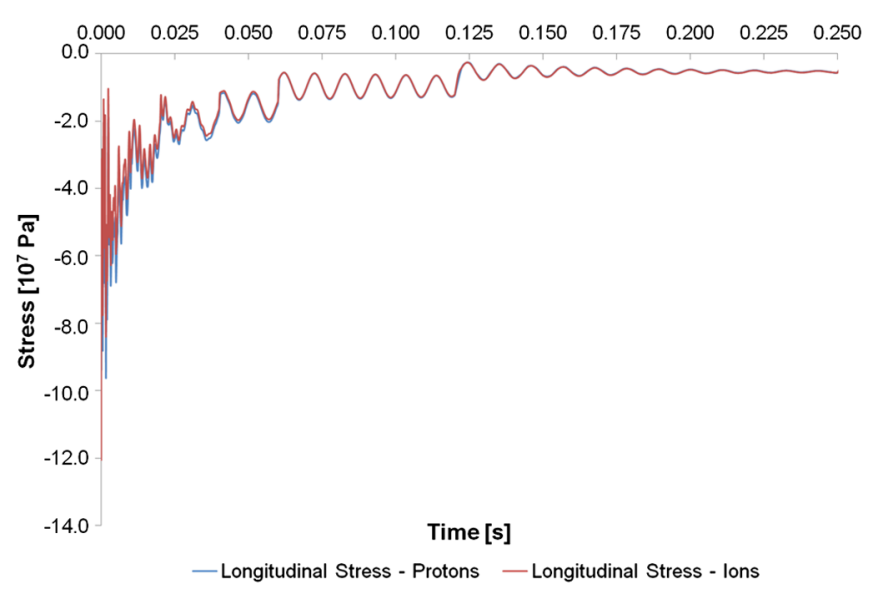

FIG. 16. Dynamic longitudinal stress (z-direction) of the collimator jaw assembly as provoked by the proton (1 bunch with $4.48 \times 10^{9}$ protons) beam impact, and as provoked by the ion (1 nominal ion bunch) beam impact. It can be observed that oscillations start at a frequency range on the order of $\mathrm{kHz}$ and settle at a main frequency of $\sim 100 \mathrm{~Hz}$, which is approximately equal to the first period of flexural oscillation of the jaw assembly. No significant difference can be observed between the proton and ion case studies.

rupture of condensed media through the nucleation, growth, and coalescence of defects due to stresses in excess of the tensile strength of the material. The beam impact generates a compressive pressure wave that is reflected as a tensile pulse from the component's free external surface. Once this tensile pulse exceeds the ultimate tensile strength (UTS) of the material, a spall is formed. Sequential stages of the damage include the appearance of micro-cracks, the coalescence of micro-cracks into one major crack and ultimately, spallation. In this study, the maximum principal stress model is used as the spallation criterion [38]. The

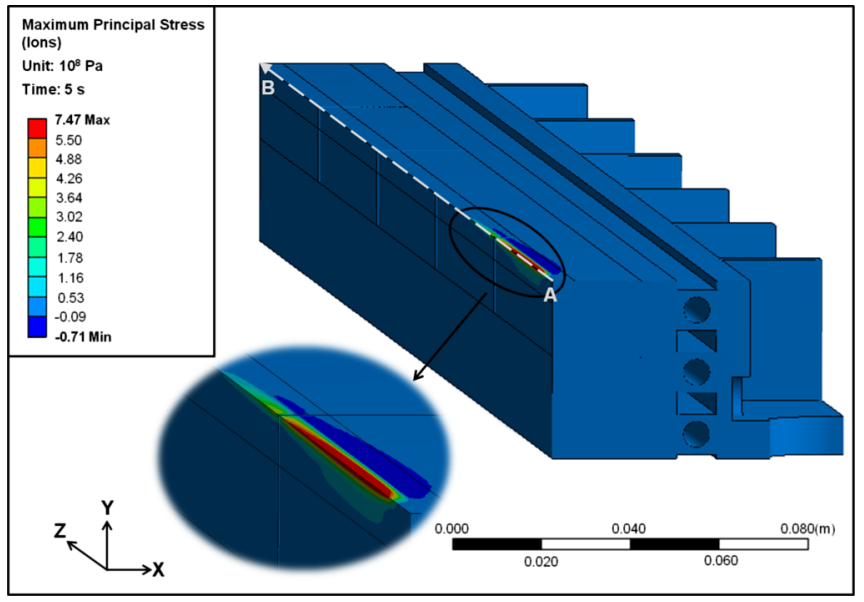

FIG. 17. Maximum principal stress on the collimator jaw assembly as provoked by the ion (1 nominal ion bunch) beam impact. The red region indicates the region of the jaw where the UTS of INERMET $^{\circledR} 180$ is exceeded, leading to the formation of micro-cracks and ultimately, spallation.

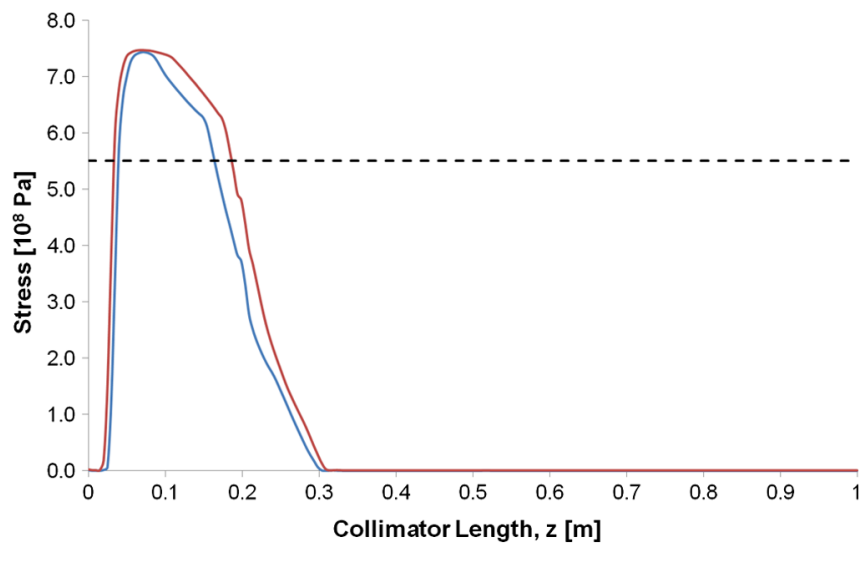

- Maximum Principal Stress - Protons - Maximum Principal Stress - Ions

- - Ultimate Tensile Strength (Inermet 180)

FIG. 18. Comparison of the maximum principal stress on the collimator jaw assembly for the proton and ion beam impacts. For each case, the maximum principal stress is plotted along Path $\mathrm{AB}$ shown in Fig. 17.

maximum principal stress on the collimator jaw for the ion case study is shown in Fig. 17 and it can observed that the highest stresses develop in the region subject to the highest temperatures (Fig. 12) and strain rates.

An overview of the UTS of INERMET ${ }^{\circledR} 180$ was obtained from experimental data [32], from which it could be concluded that under high strain rate and high temperature $\left(T_{\max } \approx 740^{\circ} \mathrm{C}\right.$ from Fig. 12$)$ conditions, the UTS of INERMET $^{\circledR} 180$ is expected to be $\sim 550 \mathrm{MPa}$. This value is thus used as a limit above which spallation is expected to occur. A comparison between the ion and proton scenarios is also given in Fig. 18 and it can be seen that the UTS of INERMET $^{\circledR} 180$ is slightly exceeded in both cases. A slightly larger region can be observed for the ion case, meaning that a slightly larger region of the first jaw insert is subject to the formation of cracks. However, it can be

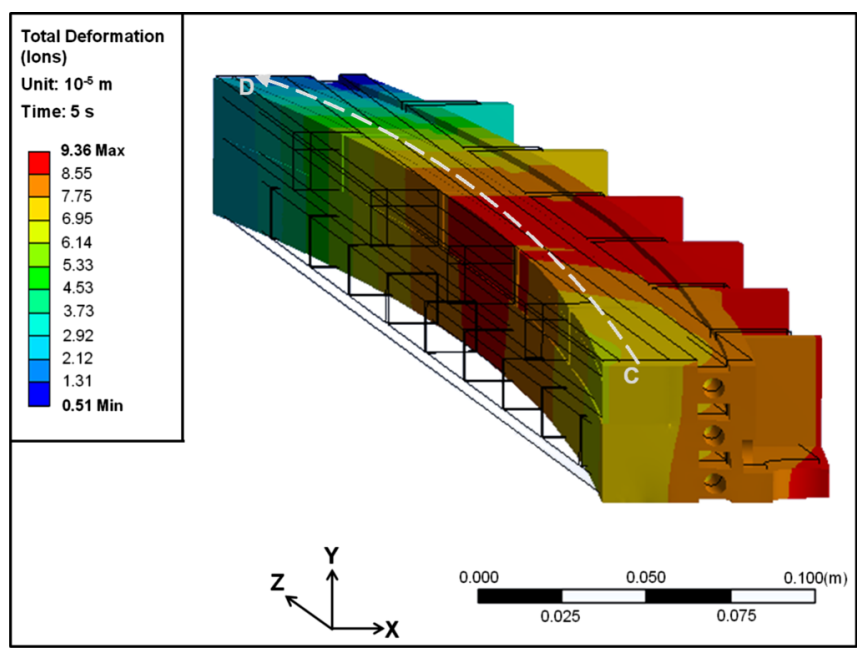

FIG. 19. Total deformation of the collimator jaw assembly as provoked by the ion (1 nominal ion bunch) beam impact. The deformation scale factor is 280 . 


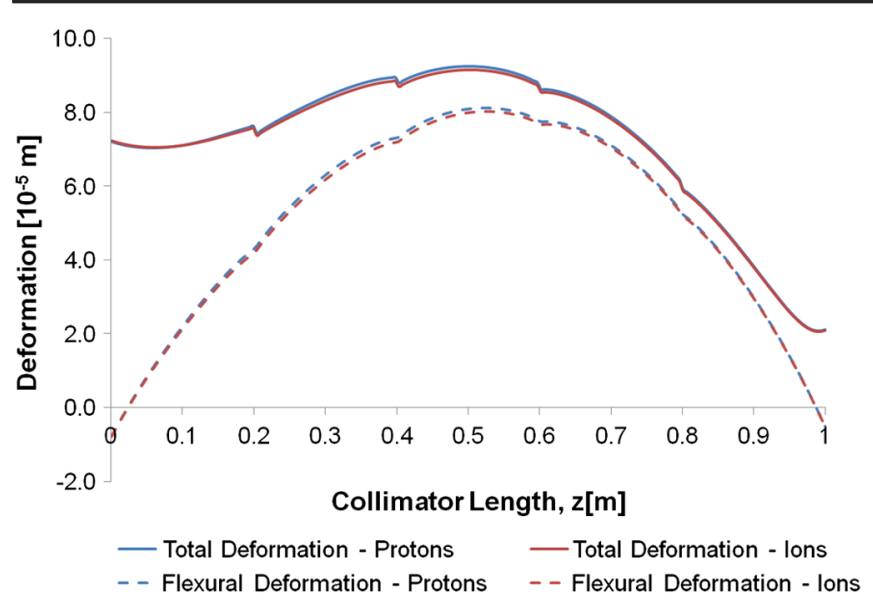

FIG. 20. Comparison of the total and flexural deformations of the collimator jaw assembly for the proton (1 bunch with $4.48 \times 10^{9}$ protons) and ion (1 nominal ion bunch) beam impacts. The deformations are plotted along Path CD shown in Fig. 19.

concluded that in both cases, crack formation and other related subsequent damage are limited to a small region.

The thermal stresses resulting from the beam impact on the collimator jaw exceed the yield strength of INERMET ${ }^{\circledR} 180$, meaning that the collimator jaw will remain subject to some permanent deformation after the beam impact. Residual plastic strains lead to a permanent deformation of $\sim 94 \mu \mathrm{m}$ of the collimator jaw assembly for both the proton and ion cases (Fig. 19, Fig. 20).

\section{CONCLUSIONS}

Predicting the consequences of high-energy particle beams impacting collimators is a fundamental issue to safely and successfully operate high-energy particle accelerators such as the LHC. The LHC is designed to bring into collision high-energy protons as well as heavy ions, and accidents involving impacts on collimators can happen in both cases. The physics of the lead ion beam's interaction with matter is qualitatively and quantitatively different from that of the proton beam, resulting in different beam-matter interaction characteristics for the two types of beam. The purpose of this study was thus to compare, for the first time, the thermomechanical response of TCTs in case of an asynchronous beam dump involving proton and heavy ion beam impacts. For both cases, the same impact conditions were assumed, changing only the particle type when simulating the interaction with matter.

A numerical FEM approach was implemented and sequential fast-transient thermostructural analyses were performed in the elastic-plastic domain in order to evaluate and compare the thermomechanical response of TCTs in case of the different beam impacts. The finite element model was implemented using recent thermophysical and structural characterization properties of the collimator jaw insert material, INERMET ${ }^{\circledR} 180$, thus providing a more reliable material model for the analyses.

From the thermal analyses, it has been shown that the heat deposition of 1 nominal ion bunch on TCTs nowhere exceeds that of 1 nominal proton bunch. However, some qualitative differences in the temperature profiles of the proton and ion beam impact case studies were observed, with such discrepancies mainly arising from ionization effects. Consequently, further detailed studies were then carried out in the structural domain to investigate if such variations in energy deposition, resulting from different beam-matter interaction processes, play any role in the mechanical behavior of the collimator structure when subject to an asynchronous beam dump. For this structural study, the proton bunch intensity was selected such that it provided the same temperature increase as a nominal ion bunch. This bunch intensity corresponded to $4.48 \times$ $10^{9}$ protons that is very close to that of an LHC pilot bunch.

The thermally induced dynamic response of the collimator structure was studied, followed by quasistatic analyses to calculate potential permanent deformations of the structure once the dynamic response had vanished. No major discrepancies were observed between the proton and ion case studies, with both having a permanent deformation of $\sim 94 \mu \mathrm{m}$ and a maximum principal stress of $\sim 745 \mathrm{MPa}$ on the collimator jaw assembly. This study thus shows that the impact of an LHC pilot proton bunch with a beam size of $0.3 \mathrm{~mm}\left(\sigma_{x}\right) \times 0.3 \mathrm{~mm}\left(\sigma_{y}\right)$ and an impact parameter of $0.5 \mathrm{~mm}$ on a TCT can be critical as it is found to cause limited irreversible damage on the TCT. Moreover, the main conclusion of this study is that heavy ion operation with a nominal ion bunch intensity of $7 \times 10^{7}$ ions and a beam size of $0.3 \mathrm{~mm}\left(\sigma_{x}\right) \times 0.3 \mathrm{~mm}\left(\sigma_{y}\right)$ poses no additional qualitative challenges, when compared to protons, on the structural integrity of collimators in case of an asynchronous beam dump.

\section{ACKNOWLEDGMENT}

This research has been supported by the European Organization for Nuclear Research (CERN, Geneva, Switzerland), the University of Malta (Malta) and the European Commission under the FP7 Research Infrastructures projects EuCARD (Grant agreement No. 227579), EuCARD-2 (Grant agreement No. 312453) and HiLumi LHC (Grant agreement No. 284404). Moreover, the authors would like to highly acknowledge the work of $\mathrm{H}$. Richter (CERN, DGS/RP) and D. Campanini (CERN, EN/MME) in developing and making available FLUKA-ANSYS interfaces. The authors are also grateful to W. Hohenauer from the Austrian Institute of Technology and M. Scapin from Politecnico di Torino for performing the measurement tests and providing the analyses of the thermal and structural characterization campaigns, respectively. 
[1] O. Brüning, P. Collier, P. Lebrun, S. Myers, R. Ostojic, J. Poole, and P. Proudlock, LHC Design Report - Volume 1, Chapter 21: The LHC as a Lead Ion Collider (CERN, Geneva, 2004), http://ab-div.web.cern.ch/ab-div/ Publications/LHC-designreport.html.

[2] D. Manglunki, M. E. Angoletta, P. Baudrenghien, G. Bellodi, A. Blas, T. Bohl, C. Carli, E. Carlier, S. Cettour-Cave, M. Chanel, K. Cornelis, H. Damerau, A. Findlay, S. Gilardoni, S. Hancock et al., Ions for LHC: performance of the injector chain, in Proceedings of the 2nd International Particle Accelerator Conference, San Sebastián, Spain (EPS-AG, Spain, 2011), p. 2529.

[3] D. Manglunki, M. E. Angoletta, H. Bartosik, G. Bellodi, A. Blas, T. Bohl, C. Carli, E. Carlier, S. Cettour-Cave, K. Cornelis, H. Damerau, I. Efthymiopoulos, A. Findlay, S. Gilardoni, S. Hancock et al., Performance of the CERN heavy ion production complex, in Proceedings of the 3rd International Particle Accelerator Conference, New Orleans, LA, 2012 (IEEE, Piscataway, NJ, 2012), p. 3752.

[4] D. Manglunki, M. E. Angoletta, H. Bartosik, G. Bellodi, A. Blas, M. Bodendorfer, T. Bohl, C. Carli, E. Carlier, S. Cettour-Cave, K. Cornelis, H. Damerau, A. Findlay, $\mathrm{S}$. Gilardoni, S. Hancock et al., The first LHC p-Pb run: performance of the heavy ion production complex in Proceedings of the 4th International Particle Accelerator Conference, IPAC-2013, Shanghai, China, 2013 (JACoW, Shanghai, China, 2013), p. 2648.

[5] R. W. Assmann, O. Aberle, A. Bertarelli, H. Braun, M. Brugger, O. Brüning, L. Bruno, S. Calatroni, E. Chiaveri, B. Dehning, A. Ferrari, B. Goddard, E. B. Holzer, J. B. Jeanneret, M. Jiménez et al., An improved collimation system for the LHC in Proceedings of the 9th European Particle Accelerator Conference, Lucerne, 2004 (EPS-AG, Lucerne, 2004), p. 536 [http://accelconf.web.cern.ch/ AccelConf/e04/].

[6] J. B. Jeanneret, D. Leroy, L. R. Oberli, and T. Trenkler, Quench Levels and Transient Beam Losses in LHC Magnets (CERN, Geneva, 1996), http://cds.cern.ch/ record/308241.

[7] O. Brüning, P. Collier, P. Lebrun, S. Myers, R. Ostojic, J. Poole, and P. Proudlock, LHC Design Report - Volume 1, Chapter 18: Beam Cleaning and Collimation System (CERN, Geneva, 2004), http://ab-div.web.cern.ch/ab-div/ Publications/LHC-designreport.html.

[8] R. Bruce, R. W. Assmann, G. Bellodi, C. Bracco, H. H. Braun, S. Gilardoni, E. B. Holzer, J. M. Jowett, S. Redaelli, and T. Weiler, Measurements of heavy ion beam losses from collimation, Phys. Rev. ST Accel. Beams 12, 011001 (2009).

[9] G. Bellodi, R. W. Assmann, R. Bruce, M. Cauchi, J. M. Jowett, G. Valentino, and D. Wollmann, First ion collimation commissioning results at the LHC, in Proceedings of the 2nd International Particle Accelerator Conference, San Sebastián, Spain (EPS-AG, Spain, 2011), p. 1813.

[10] R. W. Assmann, Collimation for the LHC high intensity beams, in Proceedings of the 46th ICFA Advanced Beam Dynamics Workshop on High-Intensity and HighBrightness Hadron Beams (HB2010), Morschach, Switzerland, (PSI, Villigen, 2010), p. 21.
[11] R. Bruce, R. W. Assmann, V. Boccone, G. Bregliozzi, H. Burkhardt, F. Cerutti, A. Ferrari, M. Huhtinen, A. Lechner, Y. Levinsen, A. Mereghetti, N. V. Mokhov, I. S. Tropin, and V. Vlachoudis, Sources of machineinduced background in the ATLAS and CMS detectors at the CERN Large Hadron Collider, Nucl. Instrum. Methods Phys. Res., Sect. A 729, 825 (2013).

[12] R. W. Assmann, I. Baishev, M. Brugger, L. Bruno, H. Burkhardt, G. Burtin, B. Dehning, C. Fischer, B. Goddard, E. Gschwendtner, M. Hayes, J. B. Jeanneret, R. Jung, V. Kain, D. Kaltchev et al., Requirements for the LHC collimation system, in Proceedings of the 8th European Particle Accelerator Conference, Paris (EPS-IGA and CERN, Geneva, 2002), p. 197.

[13] R. W. Assmann, B. Goddard, E. B. Vossenberg, and E. Weisse, The Consequences of Abnormal Beam Dump Actions on the LHC Collimation System (CERN, Geneva, 2002), https://cds.cern.ch/record/691845.

[14] R. Bruce, R. W. Assmann, F. Burkart, M. Cauchi, D. Deboy, L. Lari, S. Redaelli, A. Rossi, B. Salvachua, G. Valentino, and D. Wollmann, Collimation settings and performance in 2011 and 2012, in Proceedings of Chamonix 2012 Workshop on LHC Performance, Chamonix, France (CERN, Geneva, 2012), p. 183.

[15] L. Lari, R. W. Assmann, V. Boccone, R. Bruce, F. Cerutti, A. Rossi, V. Vlachoudis, A. Mereghetti, and A. Faus-Golfe, Accelerator physics studies on the effects from an asynchronous beam dump onto the LHC experimental region collimators, in Proceedings of $3 \mathrm{rd}$ International Particle Accelerator Conference, New Orleans, Louisiana, USA (IEEE, Piscataway, NJ, 2012), Vol. C1205201, p. 547.

[16] A. Bertarelli, V. Boccone, F. Carra, F. Cerutti, A. Dallocchio, N. Mariani, L. Peroni, and M. Scapin, Limtis for Beam Induced Damage: Reckless or too Cautious?, in Proceedings of Chamonix 2011 Workshop on LHC Performance, Chamonix, France (CERN, Geneva, 2011), p. 183.

[17] M. Cauchi, R. W. Assmann, A. Bertarelli, F. Carra, L. Lari, A. Rossi, P. Mollicone, and N. Sammut, Thermomechanical assessment of the effects of a jaw-beam angle during beam impact on Large Hadron Collider collimators, Phys. Rev. ST Accel. Beams 18, 021001 (2015).

[18] M. Cauchi, O. Aberle, R. W. Assmann, A. Bertarelli, F. Carra, K. Cornelis, A. Dallocchio, D. Deboy, L. Lari, S. Redaelli, A. Rossi, B. Salvachua, P. Mollicone, and N. Sammut, High energy beam impact tests on a LHC tertiary collimator at the CERN high-radiation to materials facility, Phys. Rev. ST Accel. Beams 17, 021004 (2014).

[19] A. Ferrari and P. R. Sala, The Physics of High Energy Reactions, in Proceedings of the Workshop on Nuclear Reaction Data and Nuclear Reactors Physics, Design and Safety, International Centre for Theoretical Physics, Miramare-Trieste, Italy, 1996, Vol. 2 (1998), p. 424.

[20] S. Eidelman, K. G. Hayes, K. A. Olive, M. AguilarBenitez, C. Amsler, D. Asner, K. S. Babu, R. M. Barnett, J. Beringer, P. R. Burchat, C. D. Carone, S. Caso, G. Conforto, O. Dahl, G. D'Ambrosio et al., Review of Particle Physics, Phys. Lett. B 592, 1 (2004).

[21] R. M. Barnett et al., Review of Particle Physics, Phys. Rev. D 54, 1 (1996). 
[22] G. Valentino, R. W. Assmann, R. Bruce, S. Redaelli, A. Rossi, N. Sammut, and D. Wollmann, Semiautomatic beam-based LHC collimator alignment, Phys. Rev. ST Accel. Beams 15, 051002 (2012).

[23] B. Goddard, R. W. Assmann, E. Carlier, J. Uythoven, J. Wenninger, and W. Weterings, Protection of the LHC against Unsynchronised Beam Aborts, in Proceedings of the 10th European Particle Accelerator Conference, Edinburgh, Scotland, 2006 (EPS-AG, Edinburgh, Scotland, 2006), p. 1514.

[24] W. Weterings, T. Antonakakis, B. Balhan, J. Borburgh, B. Goddard, C. Maglioni, and R. Versaci, Upgrade of the LHC Beam Dumping Protection Elements, in Proceedings of the 3rd International Particle Accelerator Conference, New Orleans, LA (IEEE, Piscataway, NJ, 2012), Vol. C1205201, p. 556.

[25] L. Lari, R. W. Assmann, M. Cauchi, A. Rossi, and A. Faus-Golfe, Improved robustness of the LHC collimation system by operating with a jaw-beam angle, in Proceedings of 3rd International Particle Accelerator Conference, New Orleans, Louisiana, USA (IEEE, Piscataway, NJ, 2012), Vol. C1205201, p. 553.

[26] A. Bertarelli, O. Aberle, R. W. Assmann, E. Chiaveri, T. Kurtyka, M. Mayer, R. Perret, and P. Sievers, The Mechanical Design for the LHC Collimators, in Proceedings of the 9th European Particle Accelerator Conference, Lucerne (EPS-AG, Lucerne, 2004), p. 545.

[27] A. Ferrari, P. R. Sala, A. Fassò, and J. Ranft, FLUKA: A multi-particle transport code (program version 2005) (CERN, Geneva, 2005).

[28] G. Battistoni, F. Cerutti, A. Fassò, A. Ferrari, S. Muraroi, J. Ranft, S. Roesler, and P. R. Sala, The FLUKA code: description and benchmarking, AIP Conf. Proc. 896, 31, (2007).
[29] $\operatorname{ANSYS}^{\circledR}$ Mechanical, Academic Research, Release 12.1, Help System, Workbench User's Guide (2009).

[30] Plansee GmbH, DENSIMET® and INERMET® Tungsten Alloys, http://www.plansee.com/en/Materials-Tungstenheavy-alloys-1577.htm.

[31] M. Cauchi and W. Hohenauer, Austrian Institute of Technology (AIT) and CERN Report No. CERN-ACCNOTE-2013-0023, 2013.

[32] M. Scapin, Mechanical characterization and modeling of the heavy tungsten alloy IT180, Int. J. Refract. Met. Hard Mater., 50, 258 (2015).

[33] F. J. Zerilli and R. W. Armstrong, Dislocation-mechanicsbased constitutive relations for material dynamics calculations, J. Appl. Phys. 61, 1816 (1987).

[34] D. Samantaray, S. Mandal, U. Borah, A. K. Bhaduri, and P. V. Sivaprasad, A thermo-viscoplastic constitutive model to predict elevated-temperature flow behaviour in a titanium-modified austenitic stainless steel, Mater. Sci. Eng. 526, 1 (2009).

[35] A. Dallocchio, Study of Thermo-Mechanical Effects Induced in Solids by High Energy Particle Beams: Analytical and Numerical Methods, Ph.D. thesis, Polytechnic University of Turin, Turin, 2008, https://cds.cern.ch/record/1314219/.

[36] W. Kalbreier, W. C. Middelkoop, and P. Sievers, External targets at the SPS (CERN, Geneva, 1974), https://cds.cern .ch/record/414673/.

[37] R. Courant, K. Friedrichs, and H. Lewy, On the partial difference equations of mathematical physics, IBM J. Res. Dev. 11, 215 (1967).

[38] M. Scapin, L. Peroni, and A. Dallocchio, Damage evaluation in metal structures subjected to high energy deposition due to particle beams, J. Phys. Conf. Ser. 305, 012062 (2011). 